\title{
Designing the Adaptive Tracking Controller for Uncertain Fully Actuated Dynamical Systems with Additive Disturbances Based on Sliding Mode
}

\author{
Chi Nguyen Van \\ Thai Nguyen University of Technology (TNUT), Institute of High-Technology Research and Development for Industry (IHRDI), \\ 3/2 Street, Thai Nguyen 23000, Vietnam
}

Correspondence should be addressed to Chi Nguyen Van; ngchi@tnut.edu.vn

Received 23 January 2016; Accepted 23 May 2016

Academic Editor: Lifeng Ma

Copyright (C) 2016 Chi Nguyen Van. This is an open access article distributed under the Creative Commons Attribution License, which permits unrestricted use, distribution, and reproduction in any medium, provided the original work is properly cited.

\begin{abstract}
This paper addresses the problem of adaptive tracking control for uncertain fully actuated dynamical systems with additive disturbance (FDSA) based on the sliding mode. We use the adaptive mechanism to adjust the uncertain parameters in sliding mode control law which can be switched to two modes depending on the sliding surface. By choosing appropriately the parameters in control law, the desired transient time can be obtained without effects of uncertain parameters and additive disturbances. The chattering phenomenon can be minimized by a chosen constant. This control method is applied to the angles tracking control of the twin rotor multi-input multi-output system (TRMS) which have nonlinear characteristics, the input torque disturbances and the coupling between the horizontal and vertical movements. The simulation and experimental results are presented that validate the proposed solution.
\end{abstract}

\section{Introduction}

The model of FDSA can be used to describe most of nonlinear systems in practice, for example, the robot systems, the magnetic lifting systems, the power motors, and the hydraulic pneumatic system. The FDSA model is typically EulerLagrange model with fully actuator which can be expressed in the form of a linear part of the parameters uncertainty in nonlinear systems.

In the operation process, there are some parameters varying by the time (temperature, mass of the end effectors) and the additive disturbances acting on the system (the viscous friction force, Coulomb friction force forces, cable moment, etc., which depend on the operating conditions), so it is hard to form exactly the mathematical model of the system which is very important to establish the control law. To take into account all above uncertainties, FDSA model can be used to express fully the unknown parameters and additive disturbances acting on the system. For that system, control requirement is to maintain the robustness with unknown parameters and additive disturbances.
The control methods for FDSA now are being researched including the adaptive controls based on Lyapunov, the composite adaptive controls, the control strategies using predictors, the optimal controls, the control methods using the neural networks, the passive control methods, and the sliding mode control.

For the methods based on Lyapunov and robust integral of the sign of the error (RISE), the authors in [1] proposed the adaptive control for FDSA using the RISE based on gradient adaptive update law to obtain the asymptotic stability despite general uncertain disturbances; however, Lyapunov based methods restrict the design of the adaptive update law. The materials [2] provided the first investigation of the ability to yield controller/update law modularity using the RISE feedback to compensate smooth bounded additive disturbances, the control input is composed of an adaptive feed forward term plus the RISE feedback term, and only semiglobal asymptotic stability is achieved with the region of attraction that can be made arbitrary large by increasing the control gain. In [3], the authors used a gradient based composite adaptive law in conjunction with the RISE feedback; with 
a fixed adaption gain the proposed controller yields semiglobal asymptotic stability and better tracking performance.

For the optimal control for FDSA, the first time the asymptotic tracking and convergence to an optimal controller can be obtained by a direct optimal controller developed in $[4,5]$. Specifically, direct and adaptive inverse optimal control techniques are applied to an uncertain nonlinear system to develop continuous controller; closed loop driven by that controller can track a desired trajectory while minimizing a meaningful cost. Based on [5], the semiglobal asymptotic optimality controller with a two-level architecture (the RISE controller yields a residual dynamical model by compensating for nonlinear uncertainties and the Stackelberg-based closed-loop controller minimizes cost functional for the residual hierarchical system) is developed by the authors in [6]. To ensure the asymptotic tracking, the sufficient gain conditions are derived. In [7], the authors presented the robust adaptive control of FDSA with unknown disturbances based on passive control system theorem.

For the methods using the algebra processing technique, in the paper [8] the motion/force tracking control of FDSA with affine constraints is investigated, constraint forces are successfully canceled in the dynamic equations, and then an integral feedback compensation strategy and an adaptive scheme are applied to identify the dynamic uncertainty. The proposed controller ensures that the position state of the closed-loop system tracks asymptotically the desired trajectory and the force tracking error has a controllable bound.

For the methods using feedback linearization, in $[9,10]$ the author used a reference model which is modeled by linearization of original FDSA nonlinear model to build an adaptive mechanism for compensating the effects of uncertain parameters. The adaptive controller is designed only for FDSA with uncertain parameters or additive disturbances. By using two additional controllers that use auxiliary control inputs along with the proportional derivative (PD) controller which is derived Lyapunov's second method, Dawson et al. in [11] examined the stability for the trajectory tracking problem of a robot manipulator, and the uniform ultimate boundedness property for the tracking error is yielded by this controller.

The presented methods above are designed based on Lyapunov methods with some disadvantages. First, the design of the adaptive update law is restricted by some forms, so it is difficult to satisfy the speed of the tracking problems and the convergence of the adaptive parameters; with the systems with fast dynamics it is impossible to apply. Second, only the semiglobal asymptotic tracking can be obtained. Third, the previous works focus on the separate situations: only for the uncertain parameters or only for additive disturbance inputs. The proportional gain of the adaptive controller depends on the bound of the disturbances.

The sliding mode control (SMC) is a special class of the variable-structure systems (VSSs) which is used widely for over 50 years to nonlinear systems with unknown parameters and/or additive disturbances due to the excellent robustness and fast response. SMC has been proven to be an effective control strategy for various types of real world applications such as robot manipulators, underwater vehicles, spacecrafts, electrical motors, power systems, and automotive engines. In [12-20], there are works focusing on the sliding mode control for the FDSA. The sliding mode controller for trajectory tracking is presented in [12]; the robustness to the external disturbances and uncertainties with high upper bound is achieved.

In this paper, we address the design of adaptive sliding mode control for FDSA; the adaptive mechanism is designed to compensate the uncertain parameters in the sliding mode controller. The adaptive sliding mode controller is proposed in this paper not only to keep the tracking of the outputs in the presence of the uncertain mass parameters but also to attenuate the influence of the input disturbance to the system. By using adaptive parameters and choosing appropriately controller parameters we conclude that the outputs of the FDSA controlled by the adaptive tracking control based on sliding mode will track the desired outputs for any arbitrary precision and this is the advantage of this method comparing with others. This method guarantees the global stabilization of the closed system and the control quality is good in the sense that tracking errors converge to zero not to the attractor with arbitrary small dimension. Those above all things are the contributions of this proposed method.

The paper is organized as follows. Next section deals with designing the adaptive tracking control based on sliding mode, followed by the application example for design of adaptive controller for twin rotor multi-input multi-output systems (TRMS), and last section consists of conclusions.

\section{Adaptive Tracking Control Based on Sliding Mode}

Consider the order $n$th fully actuated dynamical systems with uncertain parameters $\underline{\theta} \in R^{m}$ and additive disturbances $\eta(t) \in R^{n},\|\eta(t)\| \leq \bar{\beta}, \beta>0$, described by the following equation:

$$
D(\underline{q}, \underline{\theta}) \underline{\ddot{q}}+C(\underline{q}, \underline{\dot{q}}, \underline{\theta}) \underline{\dot{q}}+G(\underline{q}, \underline{\theta})=\underline{u}+\underline{\eta}(t),
$$

where $D=D(q, \underline{\theta}) \in R^{n \times n}$ and $C=C(q, \dot{q}, \underline{\theta}) \in R^{n \times n}$ denote the generalized inertia matrix and the centripetal-coriolis matrix, respectively, $G=G(q, \underline{\theta}) \in R^{n \times 1}$ denotes gravity vector, $\eta(t) \in R^{n}$ denotes the additive disturbances (e.g., external disturbances, unmodeled effects), and the matrixes $D(\underline{q}, \underline{\theta})$, $C(q, \dot{q}, \underline{\theta})$, and $G(q, \underline{\theta})$ depend on the uncertain parameters vector $\underline{\theta}$ (e.g., unknown parameters in the model, the slowly varying parameters in the process). $u \in R^{n}$ represents the input control vector, and $q(t), \dot{q}(t), \underline{\ddot{q}}(t) \in R^{n}$ denote the position, velocity, and acceleration vectors, respectively. The technical note $\|\cdot\|$ denotes the standard Euclidean norm for a vector or the induced infinity norm for a matrix; $|\cdot|$ denote the absolute value of the scalar argument.

Assume that (1) can be linearized and parameterized as follows:

$$
\begin{gathered}
D(\underline{q}, \underline{\theta}) \underline{\ddot{q}}+C(\underline{q}, \underline{\dot{q}}, \underline{\theta}) \underline{\dot{q}}+G(\underline{q}, \underline{\theta}) \\
=F_{0}(\underline{q}, \dot{q}, \ddot{q})+F_{1}(\underline{q}, \underline{\dot{q}}, \ddot{q}) \underline{\theta} .
\end{gathered}
$$


The desired trajectory is assumed $\underline{w}(t)$ such that $\underline{\dot{w}}(t), \underline{\ddot{w}}(t) \epsilon$ $R^{n}$ exist and are bounded and the position tracking errors denoted by $\underline{e}(t) \in R^{n}$ are defined as

$$
\underline{e}(t)=\underline{w}(t)-\underline{q}(t)
$$

and similarly, the velocity tracking errors are denoted as

$$
\underline{\dot{e}}(t)=\underline{\dot{w}}(t)-\underline{\dot{q}}(t) .
$$

The objective is to design an adaptive sliding mode controller which ensures that system (1) tracks a desired time varying trajectory $\underline{w}(t)$ despite uncertain parameters $\underline{\theta}$ in the systems and additive disturbances $\eta(t)$ acting on the dynamical model. The dimension of the attractor of tracking errors is arbitrary small; the closed loop driven by adaptive slide mode controller is global stabilization. In this paper, we propose the adaptive sliding mode controller for (1) as follows:

$$
\underline{u}= \begin{cases}\widehat{D}(K \operatorname{sign}(S)+\Gamma \underline{\dot{e}}+\underline{\ddot{w}})+\widehat{C} \dot{q}+\widehat{G} & \text { if }|S|>\varphi \\ \widehat{D}\left(K \frac{S}{\varphi}+\Gamma \underline{\dot{e}}+\underline{\ddot{w}}\right)+\widehat{C} \dot{q}+\widehat{G} & \text { if }|S| \leq \varphi,\end{cases}
$$

where $\widehat{D}, \widehat{C}$, and $\widehat{G}$ are short notations of $\widehat{D}(\underline{q}, \widehat{\hat{\theta}}), C(\underline{q}, \underline{\dot{q}}, \underline{\widehat{\theta}})$, and $G(\underline{q}, \underline{\hat{\theta}})$, respectively, and the sliding surface is formed as

$$
\underline{s}(\underline{e})=K \underline{e}+\underline{\dot{e}}
$$

and the adaptive mechanism for uncertain parameters as

$$
\frac{d \underline{\hat{\theta}}}{d t}=\left(\left(\begin{array}{c}
\Theta \\
\widehat{D}^{-1}(\underline{q}, p)
\end{array}\right) F_{1}\right)^{T} P\left[\begin{array}{l}
\underline{e} \\
\dot{e}
\end{array}\right],
$$

where $K=k I$ is a symmetric positive matrix, $k>0$ is an optional positive parameter, $\Gamma=\lambda I, \lambda>0$ is sliding parameter, $\varphi>0$ is a positive parameter, and the positive matrix $P$ is defined:

$$
P=\frac{1}{2}\left(\begin{array}{cc}
\rho I & \lambda I \\
\lambda I & I
\end{array}\right), \quad I \in R^{n \times n}, \rho>0 .
$$

The tracking errors of the closed-loop system driven by the sliding mode controller (5) and the adaptive mechanism (7) is arbitrary small by choosing the parameters $k$. The parameters $\varphi$ is used for eliminating the chattering phenomenon of the control input. The transient period of performance can be adjusted by the $\lambda$ parameter.

Theorem 1. The sliding mode controller given in (5) in conjunction with adaptive mechanism (7), where the tracking errors is generated from (3), (4), provided the control parameters $k / \varphi \geq \beta, k>0, \varphi>0, \lambda>0$ ensures that the position tracking error of the closed loop of system (1) are regulated in the sense that

$$
\|e(t)\| \longrightarrow 0, \quad t \longrightarrow \infty
$$

despite uncertain parameters $\underline{\theta}$ and additive bounded disturbances $\underline{\eta}(t)$ with $\|\underline{\eta}(t)\| \leq \beta$ acting on the system.
Proof. Let us consider the following positive definite function as a Lyapunov function candidate

$$
V(\underline{e}, \underline{\theta})=\frac{1}{2} S^{T} S+(\underline{\theta}-\underline{\hat{\theta}})^{T} Q(\underline{\theta}-\underline{\hat{\theta}})
$$

with $Q=I \in R^{m \times m}, m$ is number of uncertain parameters in vector $\underline{\hat{\theta}}$. Differentiating function $V$ with respect to time, we have

$$
\frac{d V}{d t}=S^{T} \dot{S}-2(\underline{\theta}-\underline{\hat{\theta}})^{T} \frac{d \underline{\hat{\theta}}}{d t} .
$$

Substituting the adaptive mechanism $\underline{\hat{\theta}}$ to (11) yields

$$
\frac{d V}{d t}=S^{T} \dot{S}-2\left(\underline{0}^{T},(\underline{\theta}-\underline{\hat{\theta}})^{T}\left(\widehat{D}^{-1} F_{1}\right)^{T}\right) P\left(\begin{array}{l}
\underline{e} \\
\dot{e}
\end{array}\right) .
$$

Using model (1) and controller (5), the dynamical part can be rewritten as

$$
\begin{aligned}
D \underline{q}+C \underline{\dot{q}}+G= & \widehat{D}(K \operatorname{sgn} S+\dot{S}+\underline{\ddot{q}})+\widehat{C} \dot{q}+\widehat{G} \\
& +\underline{\eta}(t),
\end{aligned}
$$

where if $S=\left(\begin{array}{lll}s_{1} & \cdots & s_{n}\end{array}\right)^{T}$ then

$$
\begin{aligned}
(\operatorname{sgn} S)^{T} S & =\left|s_{1}\right|+\cdots+\left|s_{n}\right| \geq \sqrt{\left|s_{1}\right|^{2}+\cdots+\left|s_{n}\right|^{2}} \\
& =|S| .
\end{aligned}
$$

Equation (13) is similar to the following equation:

(i) If $|S|>\varphi$,

$$
\begin{gathered}
(D-\widehat{D}) \underline{\ddot{q}}+(C-\widehat{C}) \underline{\dot{q}}+(G-\widehat{G}) \\
=\widehat{D}(K \operatorname{sgn} S+\dot{S})+\underline{\eta}(t) .
\end{gathered}
$$

From the linearized parameterized characteristic of the uncertain parameter vector and the nonsingular positive matrix $\widehat{D}$, we have

$$
\widehat{D}^{-1} F_{1}(\underline{\theta}-\underline{\hat{\theta}})=(K \operatorname{sgn} S+\dot{S})+\underline{\eta}(t)
$$

or

$$
(\underline{\theta}-\underline{\hat{\theta}})^{T}\left(\widehat{D}^{-1} F_{1}\right)^{T}=(K \operatorname{sgn} S+\dot{S}+\underline{\eta}(t))^{T} .
$$

So

$$
\begin{aligned}
\frac{d V}{d t}= & S^{T} \dot{S} \\
& -\left(\underline{0}^{T},(K \operatorname{sgn} S+\dot{S}+\underline{\eta}(t))^{T}\right)\left(\begin{array}{cc}
\rho I & \lambda I \\
\lambda I & I
\end{array}\right)\left(\begin{array}{c}
\underline{e} \\
\underline{\dot{e}}
\end{array}\right) \\
= & S^{T} \dot{S}-(K \operatorname{sgn} S+\dot{S}+\underline{\eta}(t))^{T} S \\
= & -k(\operatorname{sgn} S)^{T} S-\underline{\eta}(t)^{T} S \\
\leq & -k(\operatorname{sgn} S)^{T} S+\|\underline{\eta}(t)\| \cdot|S| \\
\leq & -k|S|+\|\underline{\eta}(t)\| \cdot|S| \leq-(k-\beta)|S| .
\end{aligned}
$$


TABLE 1: The meaning of the control parameters.

\begin{tabular}{ll}
\hline The parameters & Meanings \\
\hline$\lambda>0$ & $\begin{array}{l}\text { Adjusting the sliding surface, the transient } \\
\text { period } \\
\end{array}$ \\
$\rho>0$ & $\begin{array}{l}\text { Positive definition matrix } P \text {, adjusting the } \\
\text { convergence of adaptive mechanism }\end{array}$ \\
$\varphi$ & $\begin{array}{l}\text { Minimizing the chattering in the control input } \\
\text { Condition of stabilization for closed loop }\end{array}$ \\
\hline
\end{tabular}

Thus, if $k>\beta$ then $d V / d t<0$ with all $|S| \neq 0$.

(ii) If $|S| \leq \varphi$,

the expression of $d V / d t$ given by (18) can be rewritten as follows:

$$
\begin{aligned}
\frac{d V}{d t} & =-k\left(\frac{S}{\varphi}\right)^{T} S-\underline{\eta}(t)^{T} S \\
& \leq-k\left(\frac{S}{\varphi}\right)^{T} S+\|\underline{\eta}(t)\| \cdot|S| \leq-\left(\frac{k}{\varphi}-\beta\right)|S| .
\end{aligned}
$$

So if $k / \varphi \geq \beta$ then $d V / d t<0$ with all $|S| \neq 0$. Therefore, $d V / d t<0$ if the parameters $k, \varphi$ are chosen such that $k / \varphi \geq$ $\beta$; then $k>\beta$; we always have $d V / d t<0$ with all $|S| \neq 0$; this mean that the closed-loop system is asymptotic stabilization with sliding mode controller (5) and the parameter adaptive mechanism (7).

With the positive matrix $P$, the tracking errors of the closed loop system are regulated to reach the sliding surface $\underline{s}(\underline{e})=\underline{0}$ by controller (5) and outputs of system (1) then converge to the desired set points. The chattering problem of the control input caused by keeping the state trajectory on the sliding surface $\underline{s}(\underline{e})=\underline{0}$ is minimized by the choosing the parameter $\varphi$, and the larger $\varphi$ is, the smaller chattering is. To adjust the performance of the closed loop, the parameters of controller (5) and adaptive mechanism (7) can be chosen appropriately; see Table 1.

\section{Application Example}

The TRMS system is manufactured by Feedback Instrument as shown in Figure 1. The TRMS model is used to test the control law in the laboratory; the important application of the TRMS model is experiments of control problems for the helicopter [21] because it is an experimental setup that resembles the helicopter model. TRMS is a fully actuated mechanical system with two links, a horizontal link connected to the tower through a pivot and a link which is perpendicular to the horizontal link connected through another rotational joint with propellers attached at both ends. TRMS is a nonlinear system including the vertical and horizontal movements which are driven by the propulsive forces due to the main rotor and the horizontal tail rotor, respectively, and the propulsive forces can be changed by the voltages applied to the DC motors. The yaw and the pitch

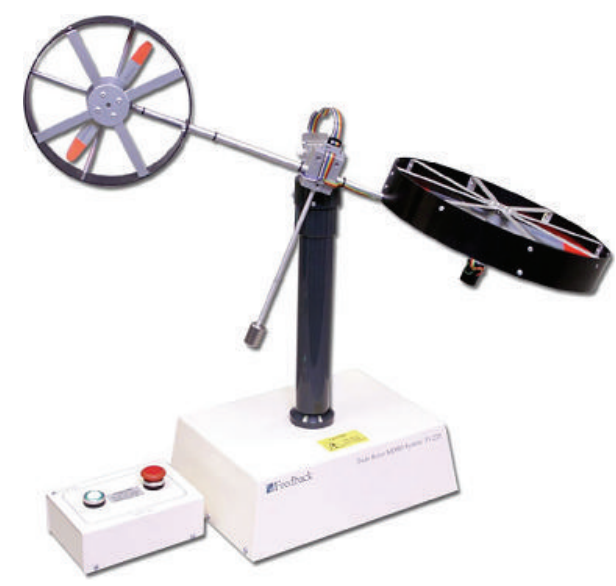

FIgure 1: The TRMS setup in the Instrument and Control Lab of Thai Nguyen University of Technology.

angles are measured by tachometers. The angle stabilization control problem for TRMS is difficult because the dynamic characteristics of TRMS, high nonlinear systems with high coupling between the horizontal motion and vertical motion, the friction moment, the cable moment, and gyro moment influence the propulsive moments as input disturbances which cannot be modeled exactly in the practice. As the rotor speeds are varying, high amount of cross coupling creeps into the system which no longer keeps systems flat [16].

This part applied the sliding mode control to design the adaptive controller for stabilizing yaw and pitch angles for TRMS. Firstly, the mathematical model of TRMS is rewritten in Euler-Lagrange forced model with uncertain parameters and input disturbances that are, respectively, the energies which depended on the mass of TRMS' parts and the friction force, the flat cable force, the effects of the speed of the main rotor on the horizontal movement, and the speed of tail rotor to the vertical movements. Then, we propose the new adaptive mechanism to adjust the parameters of dynamic model in sliding mode control law to compensate the influences of the uncertain parameters and input disturbances to the tracking errors; the potential energies are selected as adaptive parameters of the adaptive mechanism. The outputs of the controller are the rotation speeds of two DC motors which are the desired set points of the inner control loop by the input voltages applied to the DC motors. By choosing appropriately adaptive controller parameters, the effects of the input disturbances to the yaw and pitch angles will be attenuated.

3.1. The Model of TRMS in Uncertain Fully Actuated Dynamical Systems with Additive Disturbances. Accurate modeling of the system is very important for developing the control law for TRMS. Authors in the [22] presented the dynamic model of TRMS using the Lagrangian method which took all the effective forces into account. Now, we consider the horizontal and vertical angles (measured outputs) denoted by $\alpha_{h}, \alpha_{v}$, the rotational speeds of tail rotor, and main rotor denoted by $\omega_{h}$, 


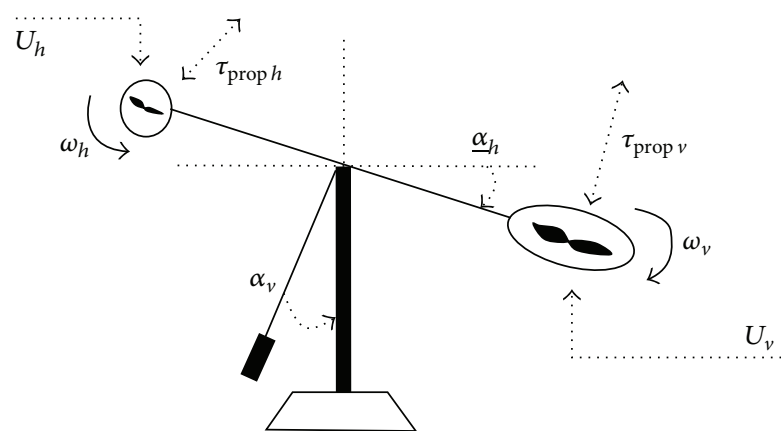

FIGURE 2: The denotations of TRMS used in the model formulations. $\omega_{v}$. The parameters $k_{f h n}, k_{f v p}, k_{f v n}, J_{1}, J_{2}, J_{3}, m T_{1}, l_{T_{1}}, m T_{2}$, $l_{T_{2}}, g, h, l_{\mathrm{t}}, l_{\mathrm{m}}$, and $k_{m}, k_{g}, k_{f h p}$, are the physical parameters and defined parameters of the TRMS listed in Figure 2 and the Appendix of this paper.

From [22], the model of TRMS is rewritten in EulerLagrange forced model as follows:

$$
D_{0}(\underline{\alpha}) \underline{\ddot{\alpha}}+C(\underline{\alpha}, \underline{\dot{\alpha}}) \underline{\dot{\alpha}}+G(\underline{\alpha})=\Im
$$

where $\underline{\alpha}=\left[\begin{array}{ll}\alpha_{h} & \alpha_{v}\end{array}\right]^{T}$ is state vector, the matrixes $D_{0}(\underline{\alpha}) \epsilon$ $R^{2 \times 2}, C(\underline{\alpha}, \underline{\dot{\alpha}}) \in R^{2 \times 2}$, and $G(\underline{\alpha}) \in R^{2 \times 1}$ are the system matrixes defined as

$$
\begin{aligned}
D_{0}(\underline{\alpha}) & =\left[\begin{array}{c|c}
J_{1} \cos ^{2} \alpha_{v}+J_{2} \sin ^{2} \alpha_{v}+h^{2}\left(m_{T_{1}}+m_{T_{2}}\right)+J_{3} & h\left(m_{T_{1}} l_{T_{1}} \sin \alpha_{v}-m_{T_{2}} l_{T_{2}} \cos \alpha_{v}\right) \\
\hline h\left(m_{T_{1}} l_{T_{1}} \sin \alpha_{v}-m_{T_{2}} l_{T_{2}} \cos \alpha_{v}\right) & J_{1}+J_{2}
\end{array}\right] \\
C(\underline{\alpha}, \underline{\alpha}) & =\left[\begin{array}{c|c}
2\left(J_{2}-J_{1}\right) \sin \alpha_{v} \cos \alpha_{v} \dot{\alpha}_{v} & h\left(m_{T_{1}} l_{T_{1}} \cos \alpha_{v}+m_{T_{2}} l_{T_{2}} \sin \alpha_{v}\right) \dot{\alpha}_{v} \\
\hline\left(J_{1}-J_{2}\right) \sin \alpha_{v} \cos \alpha_{v} \dot{\alpha}_{h} & 0
\end{array}\right] \\
G(\underline{\alpha}) & =\left[\begin{array}{c}
0 \\
\hline g\left(m_{T_{1}} l_{T_{1}} \cos \alpha_{v}+m_{T_{2}} l_{T_{2}} \sin \alpha_{v}\right)
\end{array}\right]
\end{aligned}
$$

and $\mathfrak{I}=\left[\begin{array}{lll}\sum_{i} \tau_{i h} & \sum_{i} \tau_{i v}\end{array}\right]^{T} \in R^{2 \times 1}$ with the elements $\sum_{i} \tau_{i h}$, $\sum_{i} \tau_{i v}$ are the sum of applied torques in the horizontal and vertical movements and can be summarized as

$$
\sum_{i} \tau_{i h}=\tau_{\text {prop } h}-\tau_{\text {frich }}-\tau_{\text {cable }}\left(\alpha_{h}\right)+\tau_{h v} .
$$

$\tau_{\text {proph }}=l_{t} F_{h}\left(\omega_{h}\right) \cos \alpha_{v}$ is the propulsive force due to the tail rotor, $\tau_{\text {frich }}$ implies the torque of the friction force, $\tau_{\text {cable }}\left(\alpha_{h}\right)$ refers to the torque of the flat cable force, the last term $\tau_{h v}=$ $k_{m} \dot{\omega}_{v} \cos \alpha_{v}$ of (22) represents the effect of the main propeller speed on horizontal movement:

$$
\sum_{i} \tau_{i v}=\tau_{\text {prop } v}-\tau_{\text {fric } v}+\tau_{v h}+\tau_{\text {gyro }}
$$

$\tau_{\text {prop } v}=l_{\mathrm{m}} F_{v}\left(\omega_{v}\right)$ represents the torque of propulsive force due to the main rotor, $\tau_{\text {fric } v}$ is the torque of the friction force, $\tau_{v h}=k_{t} \dot{\omega}_{h}$ denotes the effect of the tail propeller speed on vertical plane movement of the beam, and $\tau_{\text {gyro }}=$ $k_{g} F_{v}\left(\omega_{v}\right) \dot{\omega}_{h} \cos \alpha_{v}$ refers to the torque of the gyroscopic effect. The functions $F_{h}\left(\omega_{h}\right), F_{v}\left(\omega_{v}\right)$ are given by the following equations:

$$
F_{h}\left(\omega_{h}\right)= \begin{cases}k_{f h p}\left|\omega_{h}\right| \omega_{h} & \omega_{h} \geq 0 \\ k_{f h n}\left|\omega_{h}\right| \omega_{h} & \omega_{h}<0\end{cases}
$$

$$
F_{v}\left(\omega_{v}\right)= \begin{cases}k_{f v p}\left|\omega_{v}\right| \omega_{v} & \omega_{v} \geq 0 \\ k_{f v n}\left|\omega_{v}\right| \omega_{v} & \omega_{v}<0\end{cases}
$$

where $\omega_{h}, \omega_{v}$ are the rotational speed of tail and main rotor, respectively. We rewrite the matrix $D_{0}(\underline{\alpha})$ of $(20)$ as below:

$$
\begin{aligned}
D(\underline{\alpha}) \underline{\alpha} & +C(\underline{\alpha}, \underline{\dot{\alpha}}) \underline{\dot{\alpha}}+G(\underline{\alpha}) \\
& =\mathfrak{J}-\left[\frac{h\left(m_{T_{1}} l_{T_{1}} \sin \alpha_{v}-m_{T_{2}} l_{T_{2}} \cos \alpha_{v}\right) \ddot{\alpha}_{v}}{h\left(m_{T_{1}} l_{T_{1}} \sin \alpha_{v}-m_{T_{2}} l_{T_{2}} \cos \alpha_{v}\right) \ddot{\alpha}_{h}}\right],
\end{aligned}
$$

where the matrix

$$
\begin{aligned}
& D(\underline{\alpha}) \\
& =\left[\begin{array}{l|c}
J_{1} \cos ^{2} \alpha_{v}+J_{2} \sin ^{2} \alpha_{v}+h^{2}\left(m_{T_{1}}+m_{T_{2}}\right)+J_{3} & 0 \\
\hline 0 & J_{1}+J_{2}
\end{array}\right]
\end{aligned}
$$

is defined positive matrix. The model of TRMS now becomes

$$
D(\underline{\alpha}) \underline{\alpha}+C(\underline{\alpha}, \underline{\dot{\alpha}}) \underline{\dot{\alpha}}+G(\underline{\alpha})=\Im_{\text {prop }}+\tau_{d}
$$

where $\mathfrak{I}_{\text {prop }}=\left[\begin{array}{ll}\mathfrak{I}_{\text {prop } h} & \mathfrak{I}_{\text {prop } v}\end{array}\right]^{T}$ is input torque vector applied to the TRMS and $\tau_{d}$ is considered the bounded input disturbance torque vector:

$$
\tau_{d}=\left[\frac{-M_{\text {frich }}-M_{\text {cable }}\left(\alpha_{h}\right)+k_{m} \dot{\omega}_{v} \cos \alpha_{v}-h\left(m_{T_{1}} l_{T_{1}} \sin \alpha_{v}-m_{T_{2}} l_{T_{2}} \cos \alpha_{v}\right) \ddot{\alpha}_{v}}{-M_{\text {fric } v}+k_{t} \dot{\omega}_{h}+M_{\text {gyro }}-h\left(m_{T_{1}} l_{T_{1}} \sin \alpha_{v}-m_{T_{2}} l_{T_{2}} \cos \alpha_{v}\right) \ddot{\alpha}_{h}}\right]
$$

with $\left\|\tau_{d}\right\|<\beta$. 
3.2. Design the Robust Tracking Controller for Uncertain Fully Actuated Dynamical Systems with Additive Disturbances Based on Sliding Mode for TRMS. After researching carefully the dynamic model of TRMS we see that the parameters $J_{1}, J_{2}$, and $J_{3}$ which stand for the unknown parameters of TRMS and its values are not known exactly. These parameters are used for the adaptive mechanism. Suppose the unknown parameter vector

$$
\underline{\theta}=\left(\begin{array}{lll}
J_{1} & J_{2} & J_{3}
\end{array}\right)^{T}
$$

and model (20) can be written as

$$
\begin{gathered}
D(\underline{\alpha}, \underline{\theta}) \underline{\ddot{\alpha}}+C(\underline{\alpha}, \underline{\dot{\alpha}}, \underline{\theta}) \underline{\dot{\alpha}}+G(\underline{\alpha}, \underline{\theta}) \\
=F_{0}(\underline{\alpha}, \underline{\dot{\alpha}}, \underline{\ddot{\alpha}})+F_{1}(\underline{\alpha}, \underline{\dot{\alpha}}, \underline{\ddot{\alpha}}) \underline{\theta},
\end{gathered}
$$

where the matrix $F_{1}(\underline{\alpha}, \underline{\dot{\alpha}}, \underline{\ddot{\alpha}})$ is

$$
F_{1}(\underline{\alpha}, \underline{\dot{\alpha}}, \underline{\ddot{\alpha}})=\left[\begin{array}{l|l|l}
f_{11} & f_{12} & f_{13} \\
\hline f_{21} & f_{22} & f_{23}
\end{array}\right],
$$

where the elements of the $F_{1}(\underline{\alpha}, \underline{\dot{\alpha}}, \underline{\ddot{\alpha}})$ are formulated as follows:

$$
\begin{aligned}
& f_{11}=\cos ^{2} \alpha_{v} \ddot{\alpha}_{h}-2 \dot{\alpha}_{v} \dot{\alpha}_{h} \sin \alpha_{v} \cos \alpha_{v}, \\
& f_{12}=\sin ^{2} \alpha_{v} \ddot{\alpha}_{h}+2 \dot{\alpha}_{v} \dot{\alpha}_{h} \sin \alpha_{v} \cos \alpha_{v}, \\
& f_{13}=\ddot{\alpha}_{h}, \\
& f_{21}=\ddot{\alpha}_{v}+\dot{\alpha}_{h}^{2} \sin \alpha_{v} \cos \alpha_{v}, \\
& f_{22}=\ddot{\alpha}_{v}-\dot{\alpha}_{h}^{2} \sin \alpha_{v} \cos \alpha_{v}, \\
& f_{23}=0 .
\end{aligned}
$$

The model of TRMS is described in form of FDSA which is

$$
\begin{gathered}
\underbrace{D(\underline{\alpha}, \underline{\theta}) \underline{\ddot{\alpha}}+C(\underline{\alpha}, \underline{\dot{\alpha}}, \underline{\theta}) \dot{\alpha}+G(\underline{\alpha}, \underline{\theta})}_{\text {dynamical part }} \\
=\underbrace{\mathfrak{\Im}_{\text {prop }}}_{\text {input }}+\underbrace{\tau_{d}}_{\text {additive disturbances }} .
\end{gathered}
$$

So, the adaptive sliding mode controller with the adaptive mechanism for the system for the TRMS (33) based on (5) and (7) can be expressed as

$$
\begin{aligned}
& \frac{d \underline{\widehat{\theta}}}{d t}=\frac{d p}{d t}=\left(\left(\begin{array}{c}
\Theta \\
\widehat{D}^{-1}(\underline{q}, p)
\end{array}\right) F_{1}\right)^{T} P\left[\begin{array}{l}
\underline{e} \\
\dot{e}
\end{array}\right], \\
& \Im_{\text {prop }} \\
& \quad= \begin{cases}\widehat{D}(K \operatorname{sign}(S)+\Gamma \underline{\dot{e}}+\underline{\ddot{w}})+\widehat{C} \dot{q}+\widehat{G} & \text { if }|S|>\varphi \\
\widehat{D}\left(K \frac{S}{\varphi}+\Gamma \underline{\dot{e}}+\underline{\ddot{w}}\right)+\widehat{C} \underline{\dot{q}}+\widehat{G} & \text { if }|S| \leq \varphi .\end{cases}
\end{aligned}
$$

The parameter vector $\underline{\hat{\theta}}$ is adjusted in adaptive process to compensate the influences of uncertain parameters $J_{1}, J_{2}$, and $J_{3}$ and the input disturbances and external disturbances;

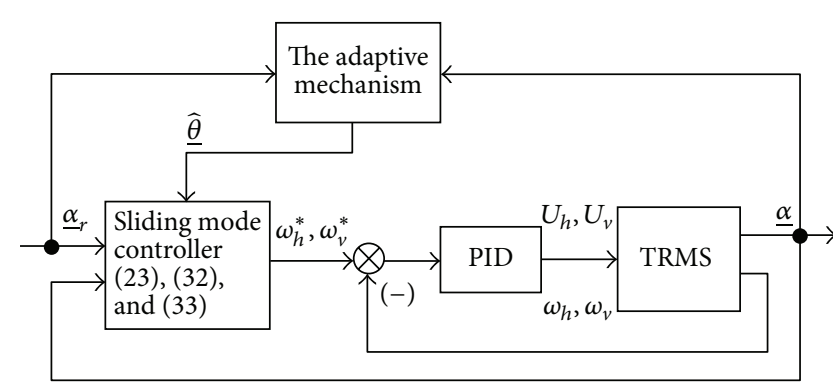

FIGURE 3: Structure of the closed-loop system with two control loops: angle control loop and rotational speed loop.

the parameter $k$ is used to adjust the sliding surface. The sliding surface vector is changed by the parameter $\lambda$; the larger $\lambda$ is, the much sloping sliding surfaces are; therefore, the tracking error tends to the origin quickly. Note that vector $\underline{\hat{\theta}}$ is not the identified vector of $J_{1}, J_{2}$, and $J_{3}$; it is used to adjust $J_{1}, J_{2}$, and $J_{3}$ in dynamical model of TRMS of sliding mode control law such that the influences of the uncertain parameters and input disturbance to the angles of TRMS are attenuated, so vector $\underline{\hat{\theta}}$ is always varied by the time. Finally, the desired rotational speed of tail and main rotor is calculated by the following equations:

$$
\begin{aligned}
& \omega_{h}^{*}= \begin{cases}\sqrt{\frac{\Im_{\text {prop } h}}{k_{f h p} \times l_{\mathrm{t}} \times \cos \alpha_{v}}} & \Im_{\text {prop } h} \geq 0 \\
-\sqrt{\frac{\left|\Im_{\text {prop } h}\right|}{k_{f h n} \times l_{\mathrm{t}} \times \cos \alpha_{v}}} & \Im_{\text {prop } h}<0,\end{cases} \\
& \omega_{v}^{*}= \begin{cases}\sqrt{\frac{\mathfrak{\Im}_{\text {prop } v}}{k_{f v p} \times l_{\mathrm{m}}}} & \Im_{\text {prop } v} \geq 0 \\
-\sqrt{\frac{\left|\mathfrak{\Im}_{\text {prop } h}\right|}{k_{f v n} \times l_{\mathrm{m}}}} & \Im_{\text {prop } v}<0 .\end{cases}
\end{aligned}
$$

From (35), the input voltages of the tail motor and the main motor can be calculated by the inner control loop. With this control loop, the proportional integral derivative (PID) controller is designed to give the input voltages $U_{h}, U_{v}$ applied to the two motors from the rotational speed errors. The structure of control system is described in Figure 3.

3.3. The Simulation and Experimental Results. In this part, we show the simulation and experimental results obtained by applying the adaptive sliding controller (34) to TRMS with physical and defined parameters listed in the Appendix. From Figures 4-12, there are the simulation results plotted by using MATLAB-Simulink R2007; in this simulation the friction torques of two channels are considered:

$$
\begin{aligned}
& \tau_{\text {frich }}=\operatorname{sign}\left(\dot{\alpha}_{h}\right)\left(0.03 \times\left|\dot{\alpha}_{h}\right|+3 \times 10^{-4}\right)(\mathrm{Nm}), \\
& \tau_{\text {fric } v} \\
& \quad=\operatorname{sign}\left(\dot{\alpha}_{v}\right)\left(0.0024 \times\left|\dot{\alpha}_{v}\right|+5.69 \times 10^{-4}\right)(\mathrm{Nm}) .
\end{aligned}
$$



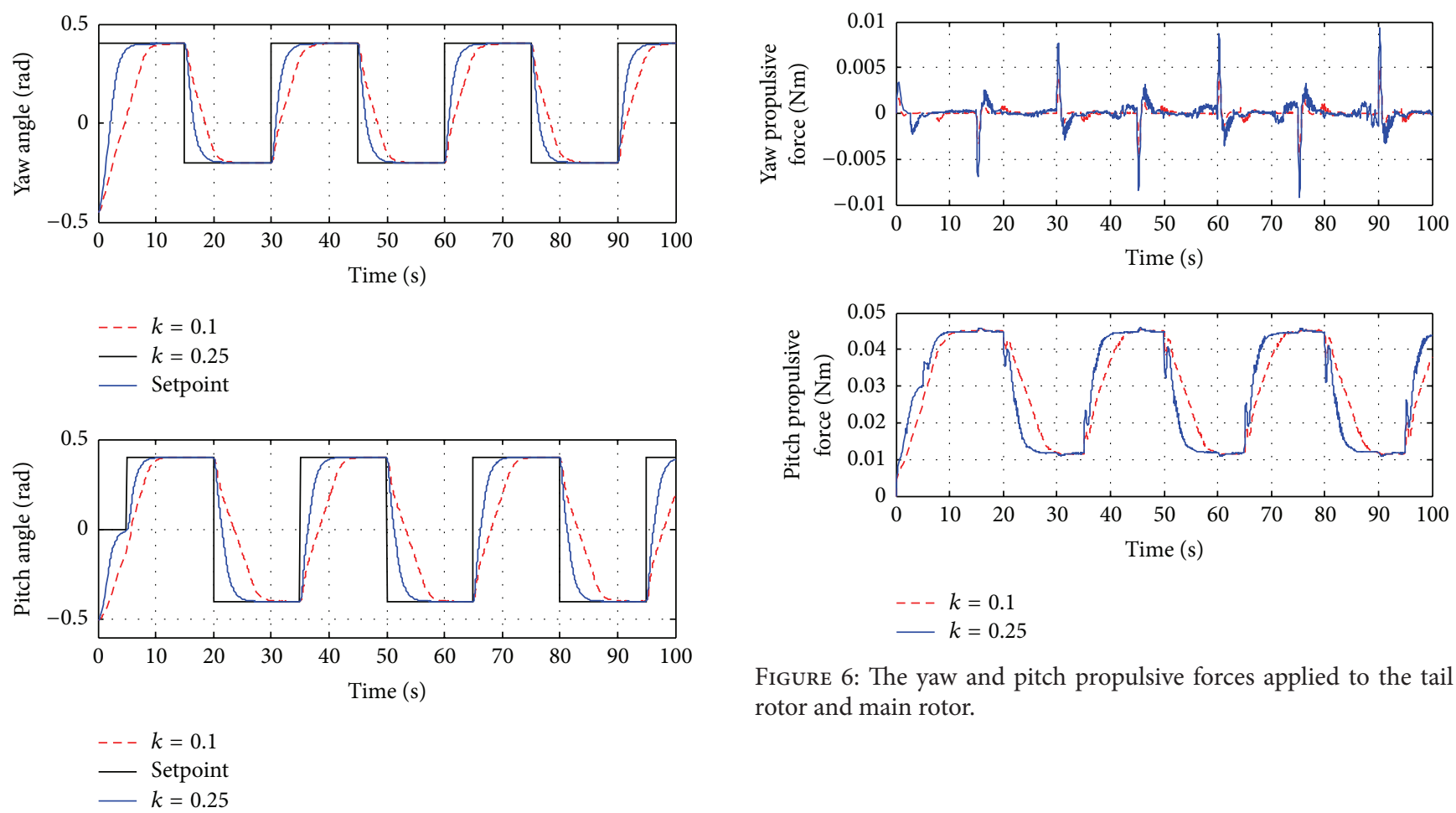

FIGURE 6: The yaw and pitch propulsive forces applied to the tail rotor and main rotor.

FIGURE 4: Simulation angle responses of yaw and pitch of TRMS controlled by adaptive controller (34) with $k=0.1$ and $k=0.25$.
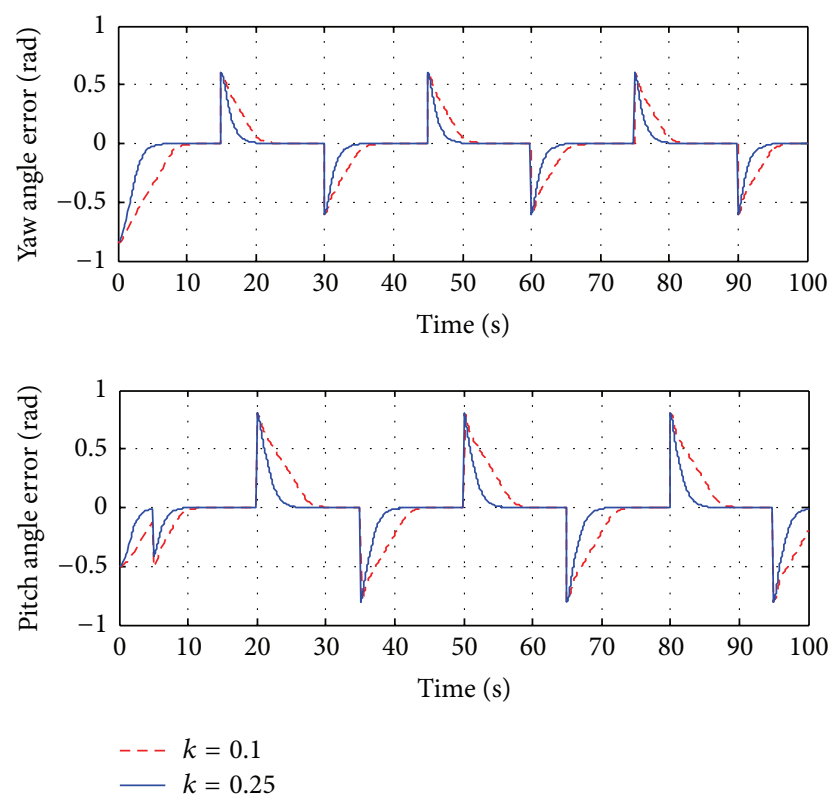

FIGURE 5: The errors of yaw and pitch angle with $k=0.1, k=0.25$.

And the cable torque is

$$
\tau_{\text {cable }}=0.0016 \times\left(\alpha_{h}+0.0002\right)(\mathrm{Nm}) .
$$

The simulation results are obtained with the choosing of $k=0.1$ and $k=0.25, \lambda=0.5, \rho=1$, and $\varphi=0.8$.
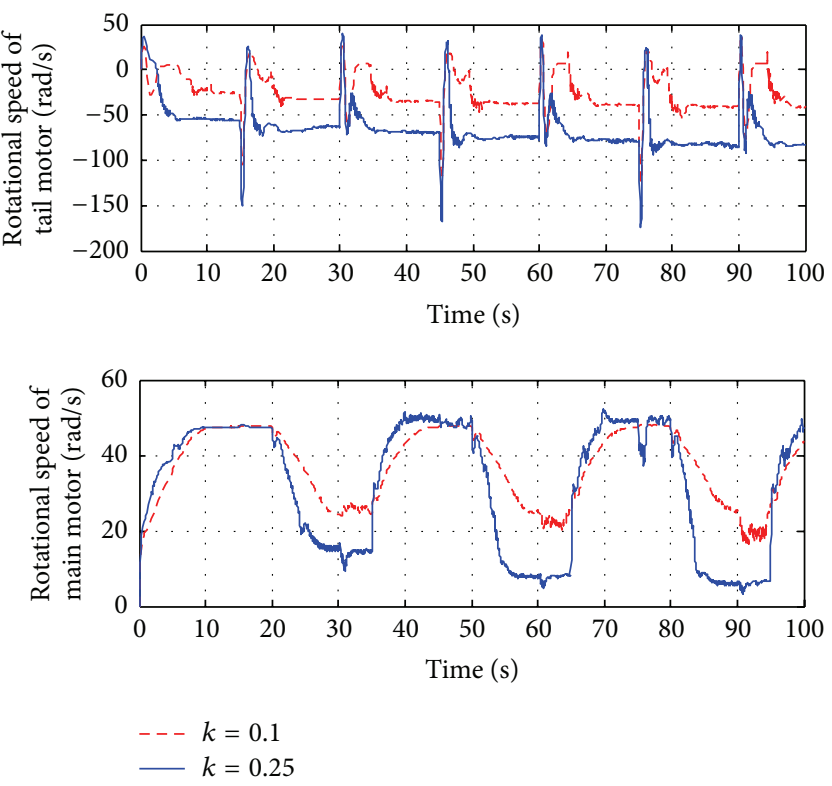

FIGURE 7: The rotational speeds of tail and main rotor.

Figure 4 shows the responses of yaw and pitch angles driven by adaptive sliding controller (34) with $\lambda=1$ and $k=0.1$ and $k=0.25$. The smaller angle errors in the static state can be kept with larger $k$.

Figures 6 and 7 represent the propulsive forces and the rotation speeds of tail and main rotor, respectively. In the yaw, there are high peaks of rotational speed of tail motor when the set point of the yaw angle changes its value with lager $k$.

In this simulation, we show the input disturbances acting on the yaw and the pitch of TRMS in Figure 8, where $\underline{\tau}_{d}$ with the bang-bang signal are caused by the sign function 

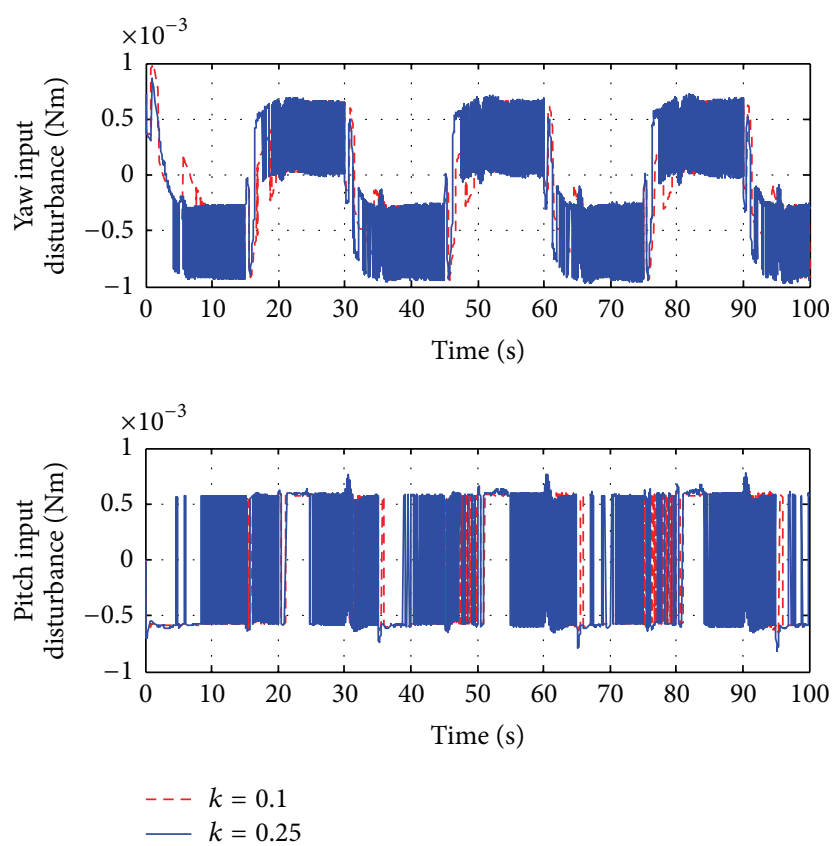

Figure 8: The yaw and pitch input disturbances $\underline{\tau}_{d}$.
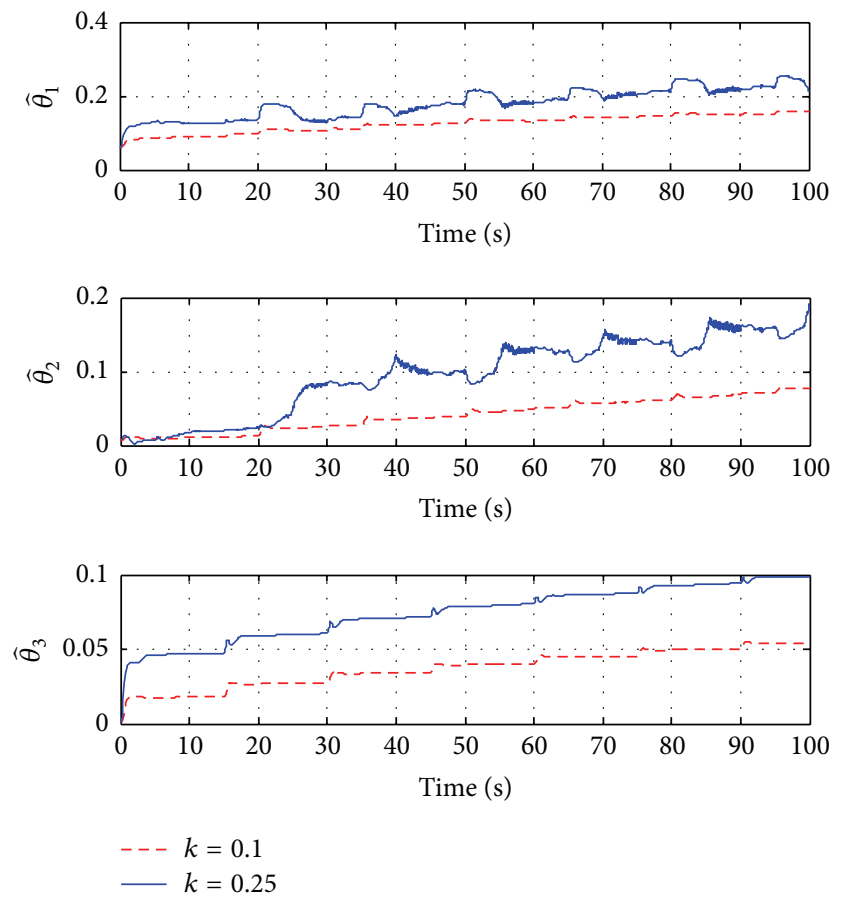

FIGURE 9: The adaptive process of parameters vector $\underline{\hat{\theta}}$.

in friction torque. Figure 9 refers to the adaptive process of parameters vector $\underline{\hat{\theta}}$. Figure 10 shows the sliding surfaces of yaw and pitch with the changing of the values of $k$ and $\lambda$. The large $\lambda$ is the shorter transient period is. Figures 11 and 12 denote the responses of yaw and pitch to the external disturbance. $\widehat{\theta}_{2}, \widehat{\theta}_{3}$ have the important role in the attenuation of external disturbance.
TABLE 2: The physical parameters supplied by the feedback instrument.

\begin{tabular}{|c|c|c|}
\hline$m_{\mathrm{b}}$ & Mass of the counterweight beam & $0.022 \mathrm{~kg}$ \\
\hline$m_{\mathrm{cb}}$ & Mass of the counterweight & $0.068 \mathrm{~kg}$ \\
\hline$m_{\mathrm{m}}$ & Mass of main part of the beam & $0.014 \mathrm{~kg}$ \\
\hline$m_{\mathrm{mr}}$ & Mass of the main DC motor & $0.236 \mathrm{~kg}$ \\
\hline$m_{\mathrm{ms}}$ & Mass of the main shield & $0.219 \mathrm{~kg}$ \\
\hline$m_{\mathrm{t}}$ & Mass of the tail part of the beam & $0.015 \mathrm{~kg}$ \\
\hline$m_{\mathrm{tr}}$ & Mass of the tail DC motor & $0.221 \mathrm{~kg}$ \\
\hline$m_{\mathrm{ts}}$ & Mass of the tail shield & $0.119 \mathrm{~kg}$ \\
\hline$r_{\mathrm{ms}}$ & Radius of the main shield & $0.155 \mathrm{~m}$ \\
\hline$r_{\mathrm{ts}}$ & Radius of the tail shield & $0.1 \mathrm{~m}$ \\
\hline$h$ & $\begin{array}{l}\text { Length of the offset between base } \\
\text { and joint }\end{array}$ & $0.06 \mathrm{~m}$ \\
\hline$g$ & Gravitational acceleration & $9.8 \mathrm{~m} / \mathrm{s}^{2}$ \\
\hline$k_{g}$ & Gyroscopic constant & 0.2 \\
\hline$k_{m}$ & Positive constant & $2 \times 10^{-4}$ \\
\hline$k_{t}$ & Positive constant & $2.6 \times 10^{-5}$ \\
\hline$l_{\mathrm{t}}$ & Length of tail part of the beam & $0.282 \mathrm{~m}$ \\
\hline$l_{\mathrm{m}}$ & Length of main part of the beam & $0.246 \mathrm{~m}$ \\
\hline$l_{\mathrm{b}}$ & Length of counterweight beam & $0.29 \mathrm{~m}$ \\
\hline$l_{\mathrm{cb}}$ & $\begin{array}{l}\text { Distance between counterweight } \\
\text { and joint }\end{array}$ & $0.276 \mathrm{~m}$ \\
\hline$k_{f h p}$ & Positive constant & $1.84 \times 10^{-6}$ \\
\hline$k_{f h n}$ & Positive constant & $2.2 \times 10^{-7}$ \\
\hline$k_{f v p}$ & Positive constant & $1.62 \times 10^{-5}$ \\
\hline$k_{f v n}$ & Positive constant & $1.08 \times 10^{-5}$ \\
\hline
\end{tabular}

To validate the performance of the controller, the experimental system is depicted in Figure 13; to obtain the response of the TRMS we use the DSP 1103 PPC controller board supplied by dSPACE; control algorithm is installed in the computer with MATLAB/Simulink R2007; after compiling, the control file is transferred to the DSP 1103 and angles of TRMS are monitored by Control Desk software.

Figure 14 shows the responses of the angles of the TRMS in experimental results with step desired angles. The controller responds quickly in attenuating the disturbance with $k=2.0$ and $\lambda=0.5, \rho=0.01$, and $\varphi=0.8$. Figure 15 depicts the control signals; there are the propulsive forces due to the main rotor and tail rotor. The chattering phenomenon in control signals is minimized by choosing $\varphi=0.8$.

\section{Conclusions}

This paper introduces the designing of the adaptive tracking control for FDSA based on the sliding mode. By using the adaptive mechanism to adjust the uncertain parameters and choosing appropriately the parameters in control law, the desired outputs of FDSA can be obtained without effects of uncertain parameters and the additive disturbances. This proposed method is applied to TRMS with angle control problem. In order to design the controller, the mathematical 


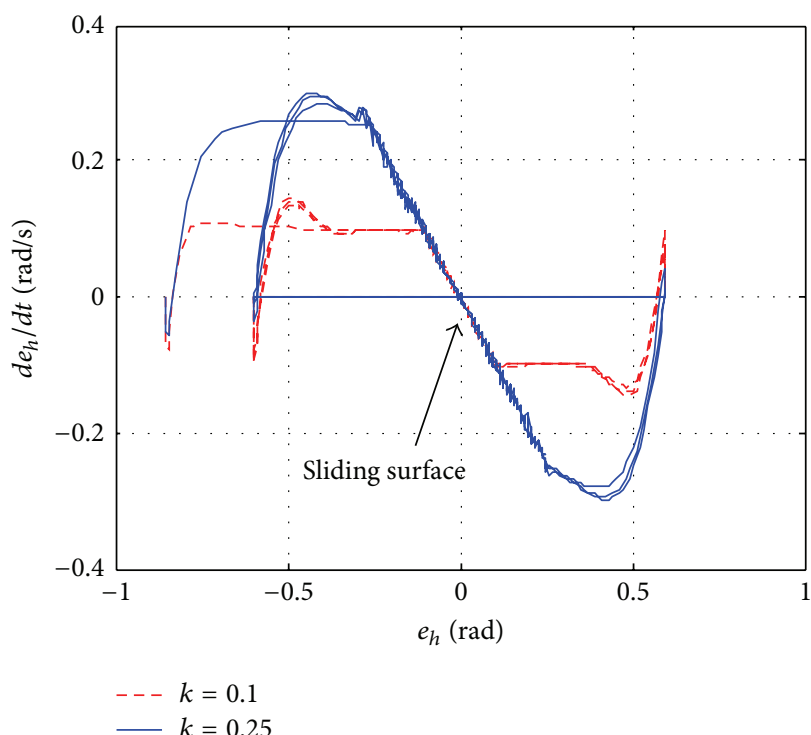

(a)

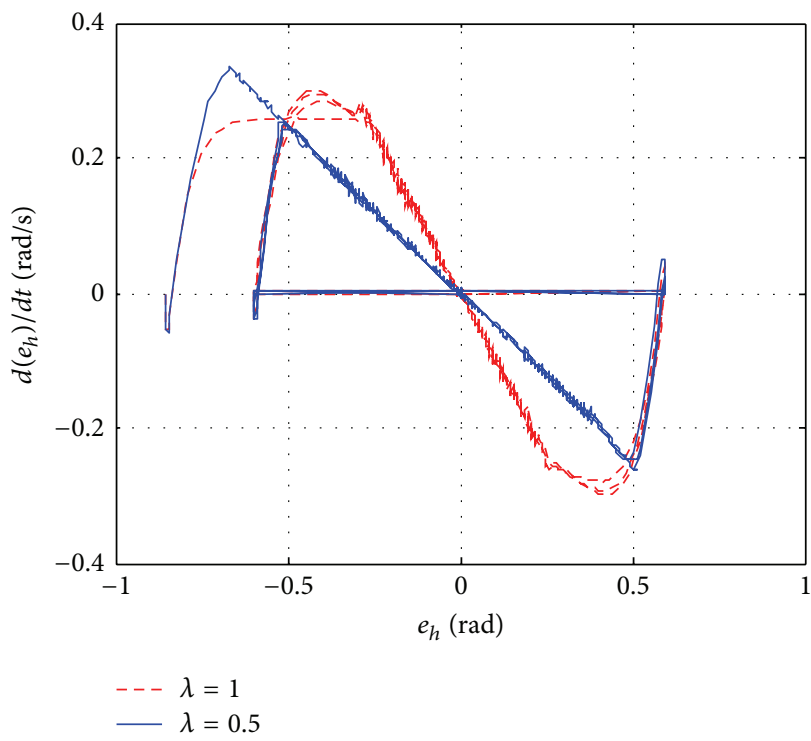

(c)

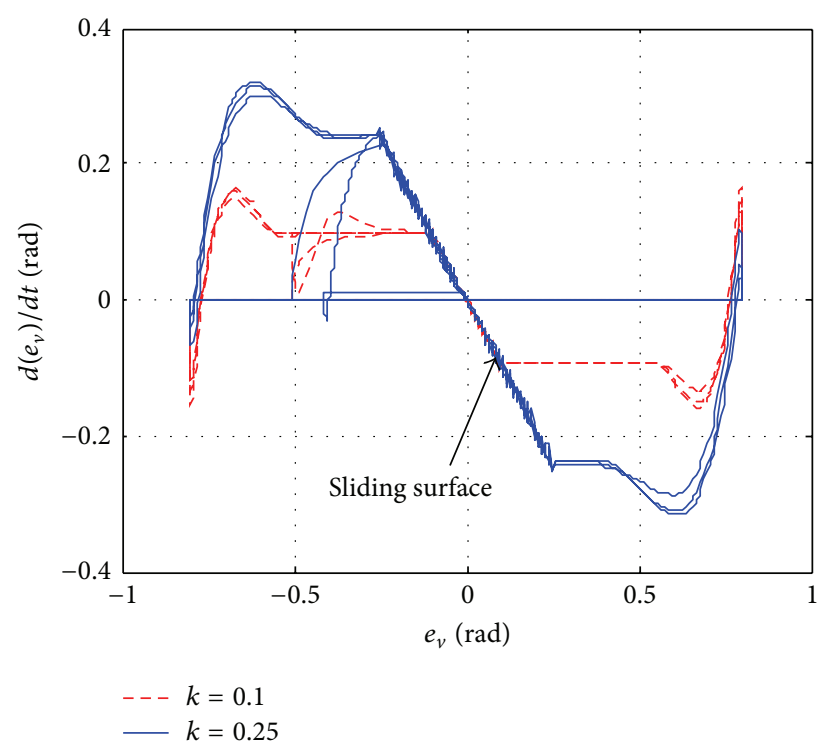

(b)

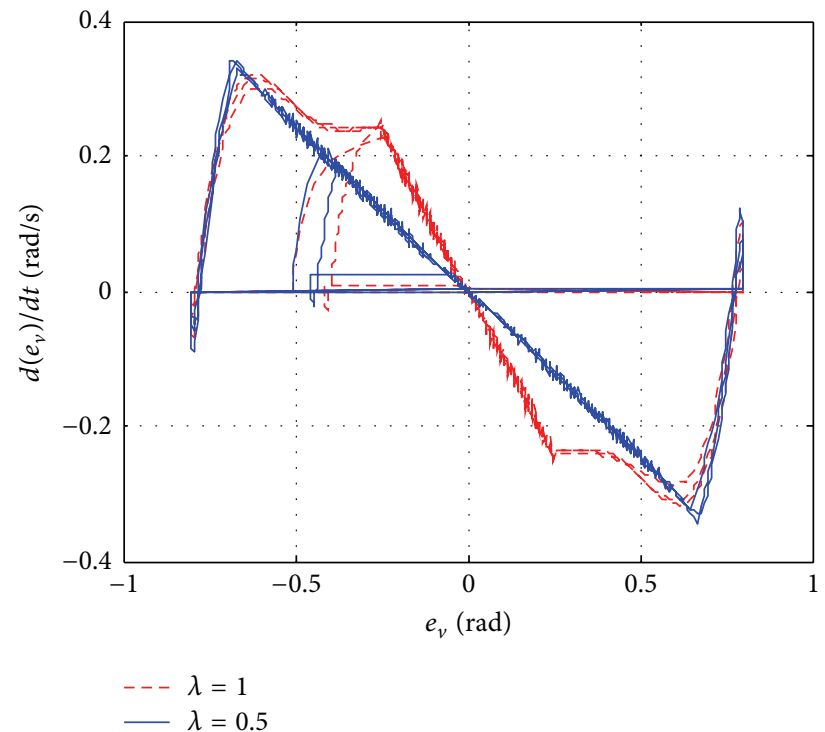

(d)

FIGURE 10: The sliding surfaces of yaw and pitch in the cases: (a) and (b) $\lambda=1, k=0.1$, and $k=0.25$; (c) and (d) $k=0.25, \lambda=1$, and $\lambda=0.5$.

model of TRMS is rewritten in FDSA form. By researching carefully the model, we consider that the energies which depended on the mass of TRMS' parts are uncertain parameters; the flat cable force, the effects of the speed of the main rotor on the horizontal movement, and the speed of tail rotor to the vertical movements are the input disturbances acting on the inputs of TRMS. The adaptive controller is designed based on the sliding mode control with the bounded input disturbances. By choosing appropriately adaptive controller parameters, the effects of the input disturbances to the yaw and pitch angles will be attenuated. The yaw and the pitch angles are controlled to track the desired angles. The algorithm is simple, and the robustness of closed loop with uncertain parameters and input disturbances is shown in The Simulation and Experimental Results.

\section{Appendix}

The physical parameters supplied by the Feedback Instruments Limited and defined parameters of TRMS are listed in Table 2.

The defined parameters of TRMS model are

$$
\begin{aligned}
& m_{T_{1}}=m_{\mathrm{t}}+m_{\mathrm{tr}}+m_{\mathrm{ts}}+m_{\mathrm{m}}+m_{\mathrm{mr}}+m_{\mathrm{ms}}, \\
& m_{T_{2}}=m_{\mathrm{b}}+m_{\mathrm{cb}}, \\
& l_{T_{1}} \\
& =\frac{\left(0.5 m_{\mathrm{m}}+m_{\mathrm{mr}}+m_{\mathrm{ms}}\right) l_{\mathrm{m}}-\left(0.5 m_{\mathrm{t}}+m_{\mathrm{tr}}+m_{\mathrm{ts}}\right) l_{\mathrm{t}}}{m_{T_{1}}},
\end{aligned}
$$



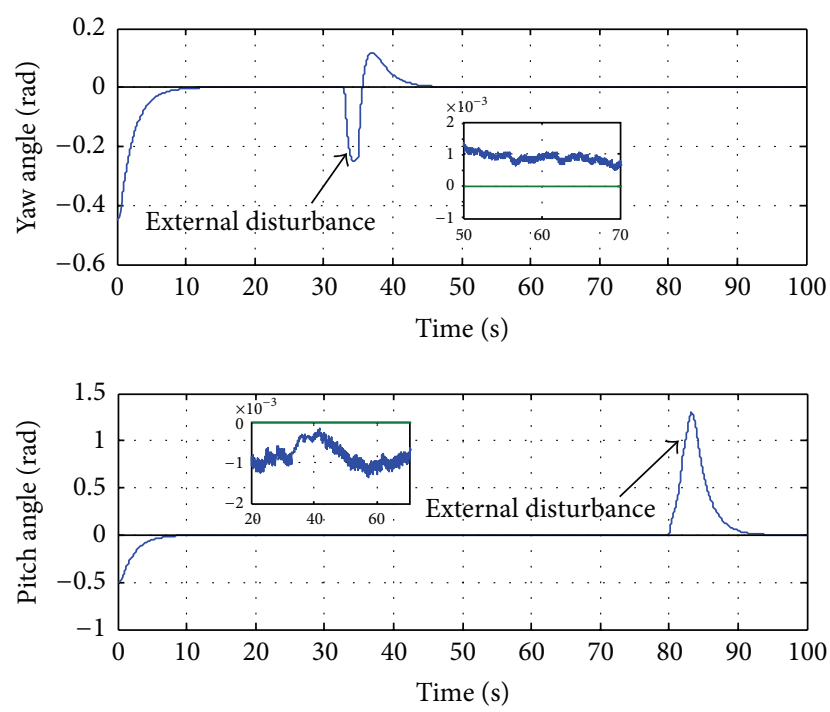

FIGURE 11: The responses of the angles with the external disturbance acting on the yaw and pitch.
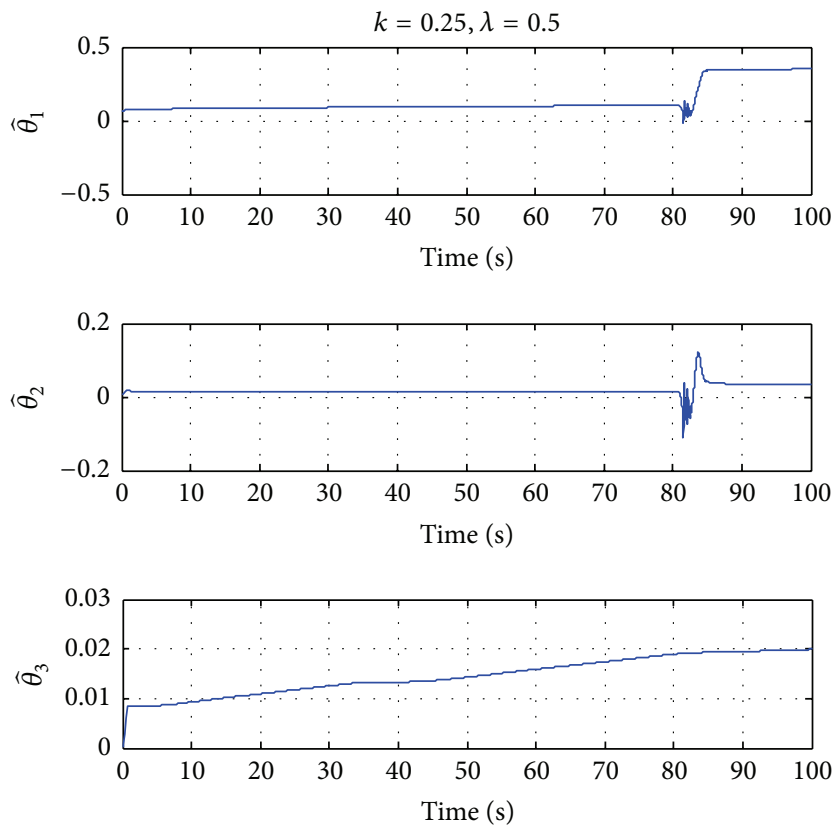

FIGURE 12: The adaptive parameters in the situation of external disturbance acting on the yaw and pitch.

$$
\begin{aligned}
& l_{T_{2}}=\frac{0.5 m_{\mathrm{b}} l_{\mathrm{b}}+m_{\mathrm{cb}} l_{\mathrm{cb}}}{m_{T_{2}}} \\
& J_{1} \\
& =\left(\frac{m_{\mathrm{t}}}{3}+m_{\mathrm{tr}}+m_{\mathrm{ts}}\right) l_{\mathrm{t}}^{2}+\left(\frac{m_{\mathrm{m}}}{3}+m_{\mathrm{mr}}+m_{\mathrm{ms}}\right) l_{\mathrm{m}}^{2} \\
& \quad+\frac{m_{\mathrm{ms}}}{2} r_{\mathrm{ms}}^{2}+m_{\mathrm{ts}} r_{\mathrm{tr}}^{2}, \\
& J_{2}=\frac{m_{\mathrm{b}}}{3} l_{\mathrm{b}}^{2}+m_{\mathrm{cb}} l_{\mathrm{cb}}^{2},
\end{aligned}
$$

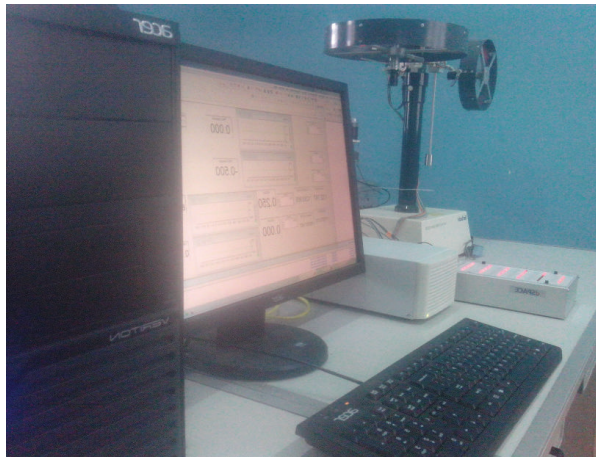

FIgURE 13: The setup of the experimental system.
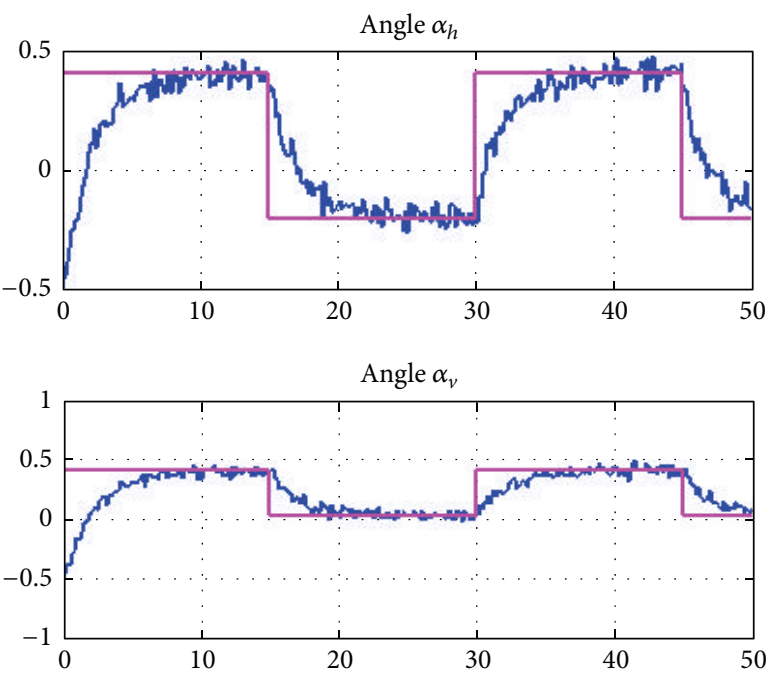

FIGURE 14: The yaw and the pitch angles.
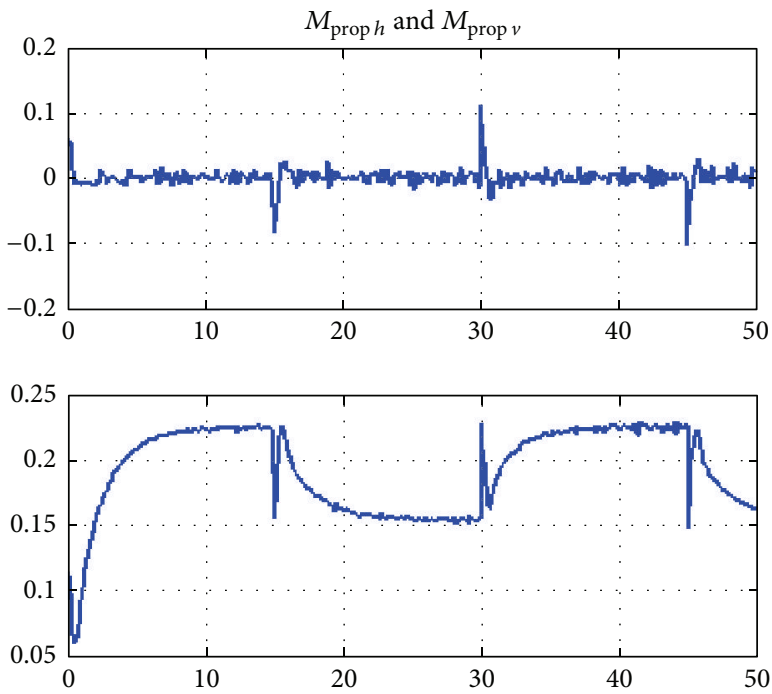

FIGURE 15: The control signals. 


\section{Competing Interests}

The author declares that they have no competing interests.

\section{References}

[1] P. M. Patre, W. MacKunis, C. Makkar, and W. E. Dixon, "Asymptotic tracking for systems with structured and unstructured uncertainties," IEEE Transactions on Control Systems Technology, vol. 16, no. 2, pp. 373-379, 2008.

[2] P. M. Patre, W. MacKunis, K. Dupree, and W. E. Dixon, "A new class of modular adaptive controllers-part I: systems with linear-in-the-parameters uncertainty," in Proceedings of the American Control Conference (ACC '08), pp. 1208-1213, Seattle, Wash, USA, June 2008.

[3] P. M. Patre, W. MacKunis, M. Johnson, and W. E. Dixon, "Composite adaptive control for Euler-Lagrange systems with additive disturbances," Automatica, vol. 46, no. 1, pp. 140-147, 2010.

[4] K. Dupree, P. M. Patre, Z. D. Wilcox, and W. E. Dixon, "Optimal control of uncertain nonlinear systems using RISE feedback," in Proceedings of the 47th IEEE Conference on Decision and Control (CDC '08), pp. 2154-2159, Cancun, Mexico, December 2008.

[5] K. Dupree, Optimal control of uncertain euler-lagrange systems [Ph.D. thesis], University of Florida, Gainesville, Fla, USA, 2009.

[6] T. Hiramatsu, M. Johnson, N. G. Fitz-Coy, and W. E. Dixon, "Asymptotic optimal tracking control for an uncertain nonlinear Euler-Lagrange system: a RISE-based closed-loop Stackelberg game approach," in Proceedings of the 50th IEEE Conference on Decision and Control and European Control Conference (CDC-ECC '11), pp. 1030-1035, IEEE, Orlando, Fla, USA, December 2011.

[7] R. Ortega, J. A. Loría Perez, P. J. Nicklasson, and H. Sira-Ramírez, Passivity-Based Control of Euler-Lagrange Systems: Mechanical, Electrical and Electromechanical Applications, Springer, London, UK, 1998.

[8] W. Sun and Y. Wu, "Adaptive motion/force control of nonholonomic mechanical systems with affine constraints," Nonlinear Analysis: Modelling and Control, vol. 19, no. 4, pp. 646-659, 2014.

[9] F. L. Levis, D. M. Dwason, and C. T. Abdalla, Robot Manipulator Control, Theory and Practice, Marcel Decker, New York, NY, USA, 2006.

[10] W. Li and J. Stoline, "Parameter estimation strategies for robotic applications," in Proceedings of the ASME Winter Annual Meeting, Boston, Mass, USA, 1987.

[11] D. M. Dawson, Z. Qu, F. L. Lewis, and J. F. Dorsey, "Robust control for the tracking of robot motion," International Journal of Control, vol. 52, no. 3, pp. 581-595, 1990.

[12] R. Solea, A. Filipescu, and U. Nunes, "Sliding-mode control for trajectory-tracking of a wheeled mobile robot in presence of uncertainties," in Proceedings of the 7th Asian Control Conference (ASCC '09), pp. 1701-1706, Hong Kong, August 2009.

[13] V. Utkin, J. Guldner, and J. Shi, Sliding Mode Control in ElectroMechanical Systems, Taylor \& Francis Group, New York, NY, USA, 1999.

[14] J.-S. Park, G.-S. Han, H.-S. Ahn, and D.-H. Kim, "Adaptive approaches on the sliding mode control of robot manipulators," Transactions on Control, Automation and Systems Engineering, vol. 3, no. 1, pp. 15-20, 2001.

[15] C. Pukdeboon, "Lyapunov optimizing sliding mode control for robot manipulators," Applied Mathematical Sciences, vol. 7, no. 61-64, pp. 3123-3139, 2013.
[16] A. K. Ekbote, N. S. Srinivasan, and A. D. Mahindrakar, “Terminal sliding mode control of a twin rotor multiple-input multiple output system," in Proceedings of the 18th IFAC World Congress, Milano, Italy, August-Septemper 2011.

[17] M. B. R. Neila and D. Tarak, "Adaptive terminal sliding mode control for rigid robotic manipulators," International Journal of Automation and Computing, vol. 8, no. 2, pp. 215-220, 2011.

[18] W. Prerruquetti and J. P. Barbot, Eds., Sliding Mode Control in Engineerring, Marcel Dekker, New York, NY, USA, 2002.

[19] T. Sun, H. Pei, Y. Pan, H. Zhou, and C. Zhang, "Neural networkbased sliding mode adaptive control for robot manipulators," Neurocomputing, vol. 74, no. 14-15, pp. 2377-2384, 2011.

[20] S. Uran and R. Šafarič, "Neural-network estimation of the variable plant for adaptive sliding-mode controller," Journal of Mechanical Engineering, vol. 58, no. 2, pp. 93-101, 2012.

[21] S. M. Ahmad, A. J. Chipperfield, and M. O. Tokhi, "Dynamic modelling and optimal control of a twin rotor MIMO system," in Proceedings of the IEEE National Aerospace and Electronics Conference (NAECON '00), pp. 391-398, Dayton, Ohio, USA, 2000.

[22] A. Rahideh and M. H. Shaheed, "Mathematical dynamic modelling of a twin-rotor multiple input-multiple output system," Proceedings of the Institution of Mechanical Engineers. Part I: Journal of Systems and Control Engineering, vol. 221, no. 1, pp. 89-101, 2007. 


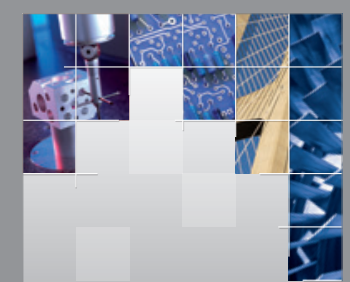

\section{Enfincering}
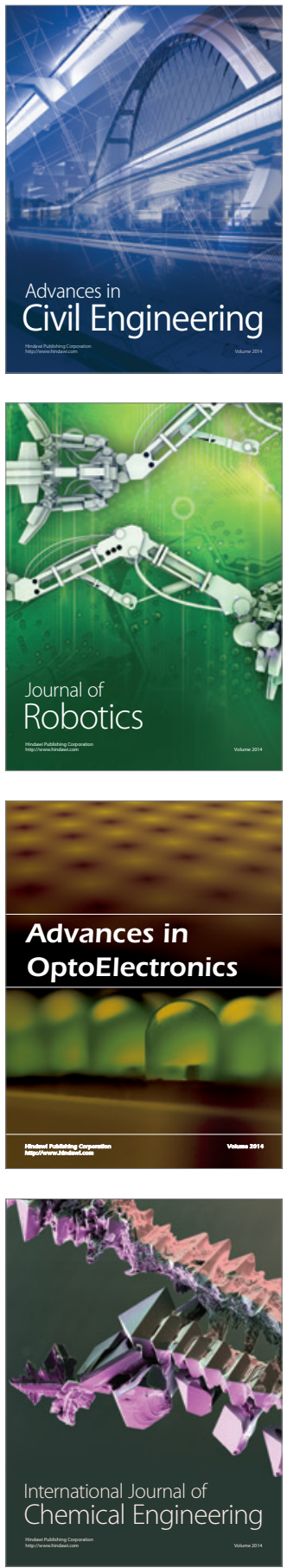

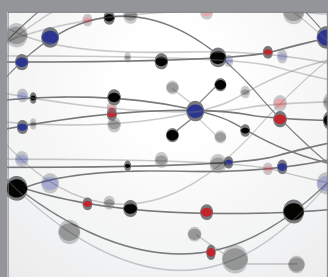

The Scientific World Journal

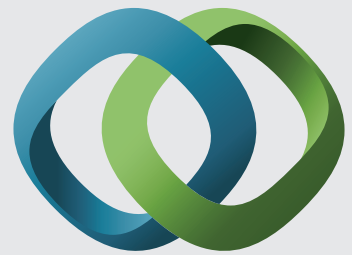

\section{Hindawi}

Submit your manuscripts at

http://www.hindawi.com
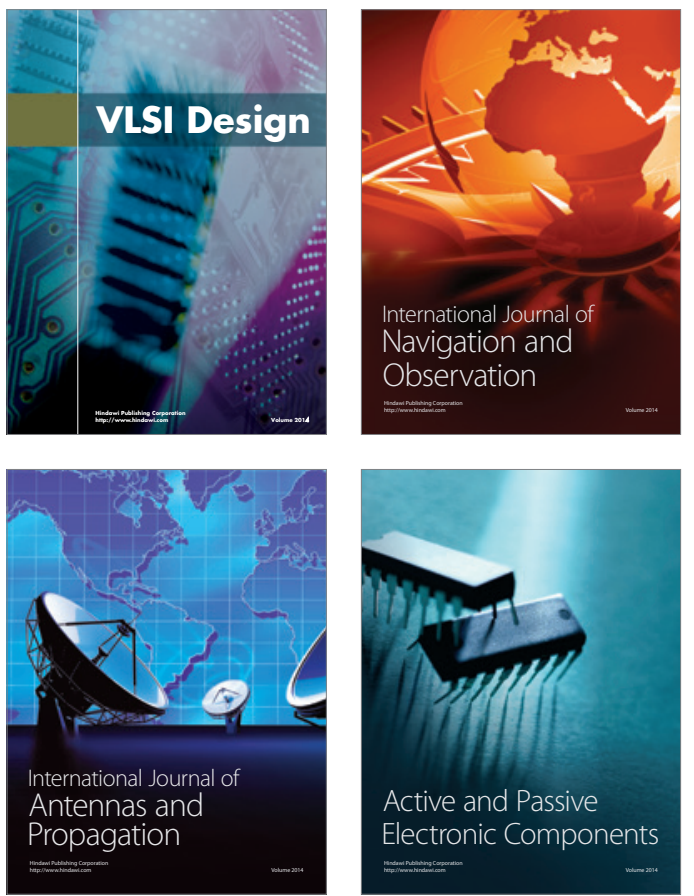
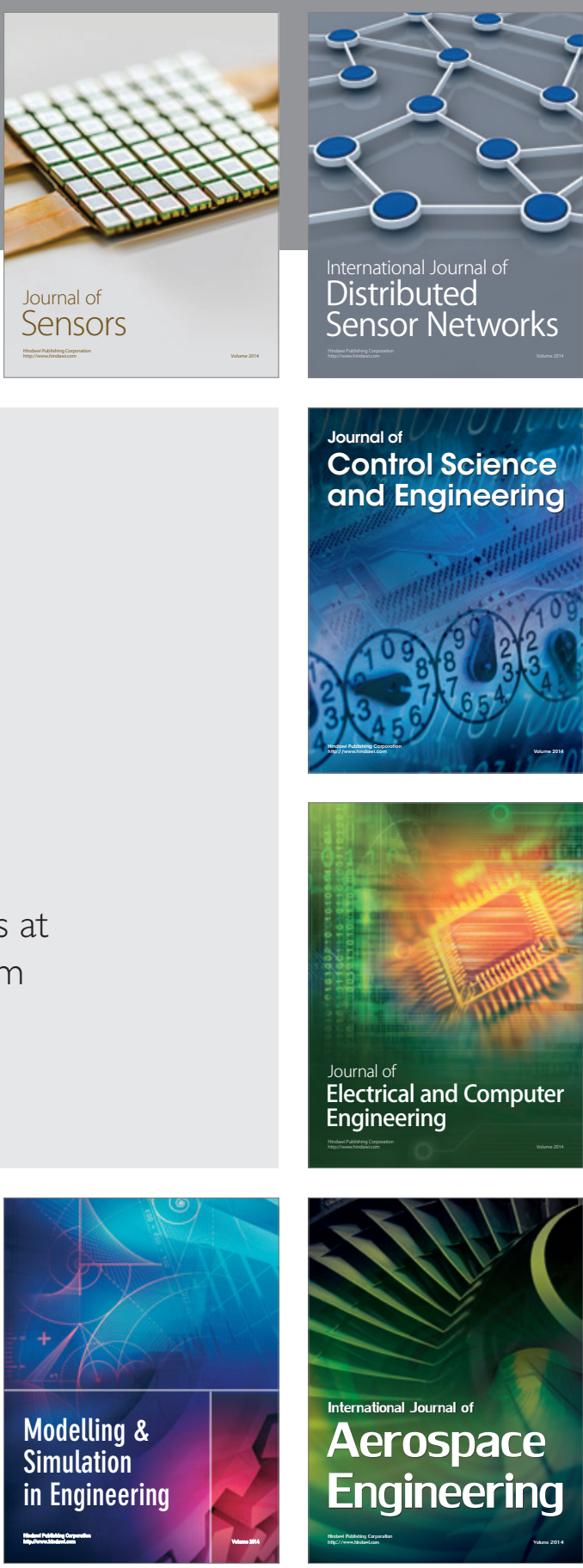

International Journal of

Distributed

Sensor Networks

Journal of

Control Science

and Engineering
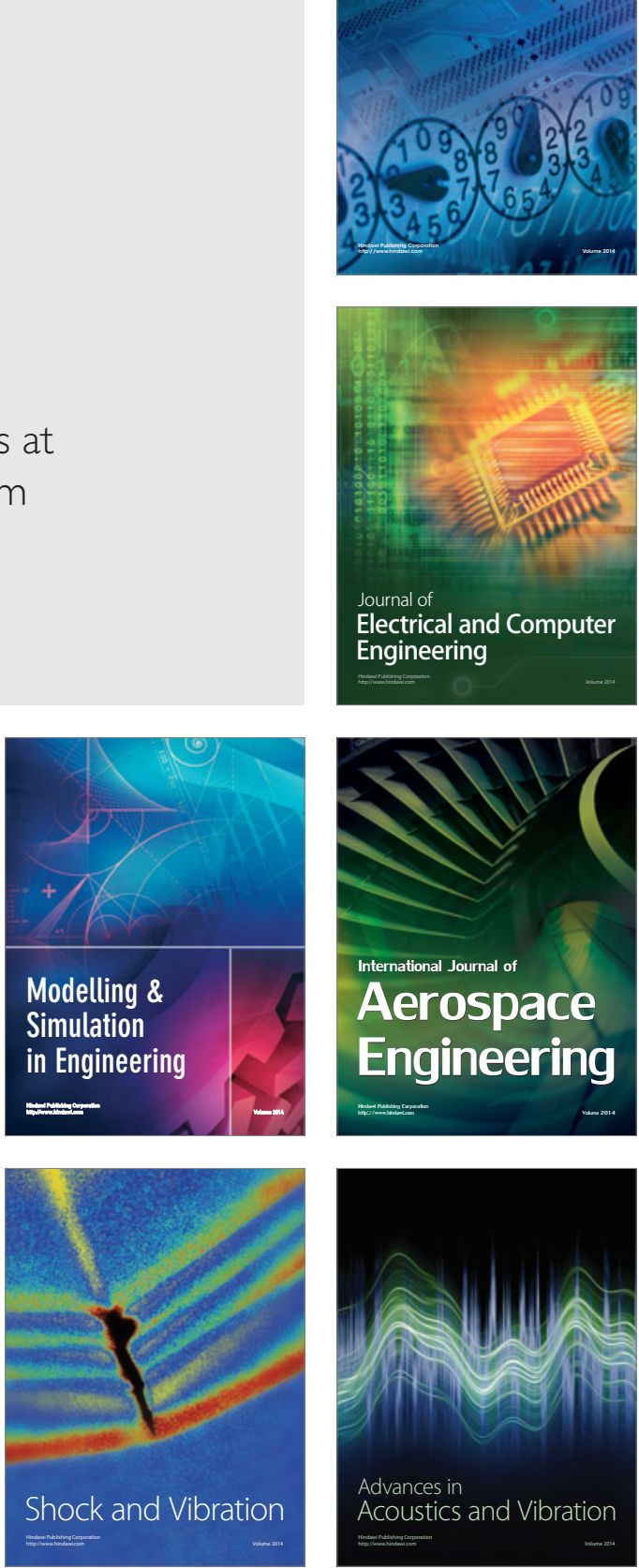